Recepción: 22 / 04 / 2019

Aceptación: 29 / 05 / 2019

Publicación: 05 / 07 / 2019

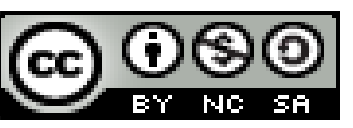

Ciencias técnicas y aplicadas

Artículo de investigación

\title{
Determinación del incremento medio anual de Tectona grandis L.F., Y Triplaris cumingiana FISCH., establecidos en sistema agroforestal con cacao (Theobroma Cacao L.)
}

\section{Determination of the average annual increase of Tectona grandis L.F., and Triplaris cumingiana FISCH., Established in an agroforestry system with cocoa (Theobroma Cacao L.)}

\section{Determinação do aumento médio anual de Tectona grandis L.F.e Triplaris cumingiana FISCH., Estabelecido em sistema agroflorestal com cacau (Theobroma Cacao L.)}

Correspondencia: cesar.cabrera@unesum.edu.ec

Cesar Alberto Cabrera-Verdesoto I cesar.cabrera@unesum.edu.ec Pedro José Suatunce-Cunuhay II pedrosuatunce@unesum.edu.ec Jorge Washington Cervantes-Borbor ${ }^{\text {III }}$ jorge.cervantes@unesum.edu.ec Mónica Virginia Tapia-Zuñiga IV monicatapia@unesum.edu.ec

I. Ingeniero Forestal. Universidad Estatal del Sur de Manabí, Docente de la Facultad de Ciencias Naturales y de la Agricultura. Carrera de Ingeniería Forestal y Agropecuaria. Campus “Los Ángeles”. Jipijapa, Manabí, Ecuador. Ingeniero Forestal, Docente de la Universidad Técnica Estatal de Quevedo, Facultad de Ciencias Ambientales. Carrera de Ingeniería Forestal, Campus "Manuel Haz Álvarez”. Jipijapa, Manabí, Ecuador.

III. Ingeniero Forestal, Universidad Estatal del Sur de Manabí Jipijapa, Manabí, Ecuador.

IV. Ingeniero Forestal. Universidad Estatal del Sur de Manabí, Facultad de Ciencias Naturales y de la Agricultura. Carrera de Ingeniería Forestal y Agropecuaria. Campus “Los Ángeles”. Jipijapa, Manabí, Ecuador. 


\title{
Resumen
}

Esta investigación se realizó en un área experimental de sistema agroforestal de teca (Tectona grandis L.f.) y Fernán Sánchez (Triplaris cumingiana F.) asociados con cacao (Theobroma cacao L.). El objetivo fue evaluar el Incremento Medio Anual (IMA) de las especies forestales, en la localidad de Quevedo. Se utilizó un diseño completamente al azar con tres repeticiones. El ensayo estuvo formado por 24 unidades experimentales, 12 para cada especie. El cacao se encuentra plantado una distancia de 3 x 3 m entre plantas e hileras. Las especies forestales fueron establecidas a una distancia de 9 × 9 m entre plantas y entre hileras. Se registró la altura en el cacao, el diámetro y la altura en las especies forestales. Los resultados obtenidos, a los cinco años de establecido el ensayo, indican que la teca obtuvo los mayores promedios de IMA en diámetro, altura, área basal y volumen. Estos resultados sugieren que las condiciones edafo-climáticas de Quevedo son apropiadas para el crecimiento de la teca.

Palabras clave: diámetro; altura; área basal; volumen.

\begin{abstract}
This research was carried out in the experiment area of agroforestry system with teak (Tectona grandis L.f.) and the ant tree (Triplaris cumingiana F.) associated with cocoa (Theobroma cocoa L.) The objective was to evaluate the Annual Increase Average (IMA) of the forest species in the location of Quevedo. A totally at random design was used with three repetition. The experiment was formed by 24 experimental units, 12 for each species. The cocoa was planted at a distance of $3 \times 3 \mathrm{~m}$ between plants and arrays. The forest species were established at a distance of $9 \times 9 \mathrm{~m}$ among plants and among arrays. The height in the cocoa, the diameter and the height in the forest species were registered. The obtained results, at the five years of the experiment established, indicate that the teak obtained the major averages of IMA in diameter and height, basal, volume. This results suggest that the conditions of soil and climate of Quevedo is appropriate for the growth of the teak.
\end{abstract}

Keys words: diameter; height; basal area; volume.

\section{Resumo}

Esta pesquisa foi conduzida em uma área experimental de sistema agroflorestal de teca (Tectona grandis Lf.) e Fernán Sánchez (Triplaris cumingiana F.) associada ao cacau (Theobroma cacao L.). O 
objetivo foi avaliar o Aumento Médio Anual (IMA) das espécies florestais, na localidade de Quevedo. Foi utilizado um delineamento inteiramente casualizado com três repetições. O ensaio consistiu de 24 unidades experimentais, 12 para cada espécie. O cacau é plantado a uma distância de 3 x 3 m entre plantas e fileiras. Espécies florestais foram estabelecidas a uma distância de 9 x $9 \mathrm{~m}$ entre plantas e entre fileiras. Altura em cacau, diâmetro e altura em espécies florestais foi registrada. Os resultados obtidos, cinco anos após o estabelecimento do ensaio, indicam que a teca obteve as maiores médias de IAM em diâmetro, altura, área basal e volume. Estes resultados sugerem que as condições edafoclimáticas de Quevedo são apropriadas para o crescimento da teca.

Palavras chaves: diâmetro; altura; área basal; volume.

\section{Introducción}

La agroforestería comenzó a atraer la atención de la comunidad científica a finales de la década de 1970; hoy en día su práctica está difundida alrededor del mundo como una de las opciones más viables para el uso sustentable de la tierra. Su potencial como sistema de producción sostenible despierta interés tanto de investigadores como de productores. La agroforestería constituye una alternativa para producción agrícola y obtención de madera al final del ciclo del sistema, lo cual incrementa la rentabilidad de una unidad de producción (Budowski, 1993).

El cultivo de cacao, principalmente en fincas de pequeños productores con áreas menores a 5 ha, normalmente se encuentra asociado con otras especies perennes; con alguna frecuencia, se establecen dentro de las plantaciones de cacao especies forestales o frutales y plátano (Musa sp.), sin seguir patrones determinados de distancia o arreglo espacial (Martínez y Enríquez 1981). En diferentes países tropicales, el cacao se establece con diferentes especies arbóreas que además de sombra aportan beneficios económicos y ecológicos complementarios al cacao (Duguma et al., 1999, Almeida et al., 2002).

En el Litoral ecuatoriano, en los últimos años, se ha incrementado el área de plantaciones forestales establecidas con especies de rápido crecimiento, tales como la teca (Tectona grandis). El uso de la teca en los proyectos de reforestación se ha incrementado por su fácil establecimiento, buen crecimiento inicial y por la calidad de la madera (Chaves y Fonseca, 2004). En investigaciones anteriores se ha obtenido buenos resultados de Fernán Sánchez asociado con cacao, por lo que se 
incluyó en este ensayo de sistema agroforestal con teca y Fernán Sánchez a fin de evaluar el crecimiento en este sistema.

En la actualidad existe gran demanda de madera de las especies tropicales, tales como: la teca y Fernán Sánchez. Estas especies tienen gran importancia, por su rápido crecimiento, su madera de muy alta calidad y gran valor comercial. La teca tiene muchos usos tales como: construcciones, ebanistería fina, chapa y contrachapada decorativa. El Fernán Sánchez también es utilizado en la ebanistería y carpintería.

Todo esto resulta favorable para establecer plantaciones comerciales, en los que se puede incluir los sistemas de plantación asociada con cultivos perennes como el cacao, ya que la demanda de teca y Fernán Sánchez en nuestro país es cada vez mayor. Además, se hace necesario realizar investigaciones sobre el crecimiento de estas dos especies establecidas en sistemas agroforestales, a fin de conocer los rendimientos de madera que obtendrán al final del turno.

\section{Materiales y Métodos}

\section{Localización y características edafo-climáticas del sitio de estudio}

la Finca Experimental "La Represa", propiedad de la Universidad Técnica Estatal de Quevedo (UTEQ), Quevedo, provincia de Los Ríos (79³0’23” O y $01^{\circ} 00^{\prime} 35^{\prime}$ ” S), ubicada en la parte alta de la cuenca del Río Guayas a $90 \mathrm{~m}$ de altitud, con características de bosque húmedo-tropical (bh-T), topografía del terreno irregular, suelo tipo Inceptisoles, textura franco arcilloso y $\mathrm{pH}$ 5.9. (INAMHI, Instituto Nacional de Meteorología e Hidrología, (2010).

\section{Metodología}

El estudio se realizó en un área experimental de sistema agroforestal de teca y fernán sánchez asociado con cacao, establecida hace cinco años. La teca y el fernán sánchez se encuentran plantados a una distancia de 9 x 9 m y el cacao a 3 x $3 \mathrm{~m}$. La metodología que se empleó en la presente investigación son los métodos: inductivo, analítico y sintético, a través de la tabulación, análisis e interpretación de los resultados obtenidos en base a los datos registrados en las parcelas de muestreo de las dos especies estudiadas. 
Esta investigación es de tipo experimental, en la que se comparó el Incremento Medio Anual (IMA) de dos especies forestales establecidas en sistema agroforestal con cacao. También se aplicó el proceso de análisis, síntesis y la inducción, mediante la inferencia estadística.

\section{Diseño experimental}

Se aplicó un diseño de bloques al azar, con tres repeticiones y dos tratamientos. Para las comparaciones de medias de los tratamientos se utilizó la prueba de " $\mathrm{t}$ " de Student al 5\% de probabilidad de error. Se utilizó el programa estadístico SAS Versión 9 (2004).

\section{Establecimiento de las unidades de muestreo (UM)}

Se establecieron un total de 24 unidades de muestreo de 18 x 18 m (324 m2), 12 unidades de muestreo para cada especie. Cada bloque estuvo conformado por cuatro unidades de muestreo. En cada parcela se registró los datos de DAP y altura total del árbol ubicado en el centro, en las dos especies forestales. También se registró la altura del cacao en los 14 árboles ubicados en el centro de cada unidad de muestreo.

\section{Resultados}

\section{Incremento en diámetro y altura de especies forestales}

El DAP y la altura total de los árboles, a los cinco años de edad, presentaron diferencias significativas entre las especies forestales $(\mathrm{P}>0,05)$. El mayor promedio en DAP y altura total fue para Tectona grandis. Por consiguiente, el IMA promedio en DAP y altura total fue mayor para Tectona. grandis (Cuadro 1).

Cuadro 1. Incremento Medio Anual (IMA) del DAP y altura total de las especies forestales asociadas con cacao, a los cinco años de edad. UTEQ, 2013.

\begin{tabular}{|l|c|c|c|c|}
\hline Especies & DAP $(\mathrm{cm})$ & IMA DAP & Altura $(\mathrm{m})$ & IMA Altura \\
\hline Teca & $18,27 \mathbf{a}$ & 3,65 & $13,75 \mathbf{a}$ & 2,75 \\
\hline Fernán Sánchez & $13,61 \mathbf{b}$ & 2,72 & $10,54 \mathbf{b}$ & 1,72 \\
\hline Error estándar & $\mathbf{1 , 8 4}$ & & $\mathbf{0 , 9 9}$ & \\
\hline
\end{tabular}

El promedio de diámetro de la teca obtenido en este estudio $(18,27 \mathrm{~cm})$ fue inferior al promedio del DAP reportado por UICYT (2002), que obtuvo un DAP de $26,5 \mathrm{~cm}$ a los cinco años de edad, en un 
ensayo de cuatro especies forestales con café. El promedio de diámetro del fernán sánchez registrado en este estudio (13,61 cm) también fue inferior al reportado por (UICYT, 2002), que alcanzó un DAP de $21,0 \mathrm{~cm}$ a los cinco años de edad.

El promedio de altura de la teca registrado en este estudio $(13,75 \mathrm{~cm})$ fue inferior al reportado por UICYT (2002), que alcanzó una altura de 16,23 m a los cinco años de edad. El promedio de altura del fernán sánchez obtenido en este estudio (10,54 m) también fue inferior al reportado por UICYT (2002), que obtuvo una altura de 15,72 $\mathrm{m}$ a los cinco años de edad.

\section{Incremento en área basal y volumen de especies forestales}

El área basal y el volumen promedio de los árboles, a los cinco años de edad, presentaron diferencias significativas entre especies forestales $(\mathrm{P}>0,05)$. El mayor promedio en área basal y volumen fue para Tectona grandis. Así mismo el mayor IMA promedio en área basal y volumen fue para Tectona grandis (Cuadro 2).

Cuadro 2. Incremento Medio Anual (IMA) del área basal y volumen de las especies forestales asociadas con cacao, a los cinco años de edad. UTEQ, 2013.

\begin{tabular}{|l|c|c|c|c|}
\hline Especies & $\begin{array}{c}\text { Área basal } \\
\left(\mathbf{m}^{\mathbf{2}}\right)\end{array}$ & $\begin{array}{c}\text { IMA } \\
\text { Área basal }\end{array}$ & $\begin{array}{c}\text { Volumen } \\
\left(\mathbf{m}^{\mathbf{3}}\right)\end{array}$ & $\begin{array}{c}\text { IMA } \\
\text { volumen }\end{array}$ \\
\hline Teca & $0,0289 \mathbf{a}$ & 0,0058 & $0,2649 \mathbf{a}$ & 0,0530 \\
\hline Fernán Sánchez & $0,0156 \mathbf{b}$ & 0,0031 & $0,0972 \mathbf{b}$ & 0,0194 \\
\hline Error estándar & $\mathbf{1 , 0 0 3 7}$ & & $\mathbf{0 , 0 3 2 4}$ & \\
\hline
\end{tabular}

El promedio del área basal de la teca obtenido en este estudio $\left(0,0289 \mathrm{~m}^{2}\right)$ fue inferior al promedio del área basal reportado por UICYT (2002), que obtuvo un área basal de $0,0558 \mathrm{~m}^{2}$ a los cinco años de edad. El promedio del área basal del fernán sánchez registrado en este estudio $\left(0,0156 \mathrm{~m}^{2}\right)$ también fue inferior al reportado por (UICYT, 2002), que alcanzó un área basal de $0,0348 \mathrm{~m}^{2}$, a los cinco años de edad.

El promedio del volumen de la teca registrado en este estudio $\left(0,2649 \mathrm{~m}^{3}\right)$ fue inferior a los reportados por UICYT (2002), que alcanzó un volumen de $0,636 \mathrm{~m}^{3}$ a los cinco años de edad. El promedio del volumen del fernán sánchez obtenida en este estudio $\left(0,0972 \mathrm{~m}^{3}\right)$ también fue inferior a los reportados por UICYT (2002), que obtuvo un volumen de $0,382 \mathrm{~m}^{3}$ a los cinco años de edad. 


\section{Altura del cacao asociado con especies forestales}

La altura del cacao, a los cinco años de edad, no presentó diferencias significativas entre las asociaciones $(\mathrm{P}<0,05)($ Cuadro 3$)$.

Cuadro 3. Altura del cacao asociado con especies forestales, a los cinco años de edad. UTEQ, 2013.

\begin{tabular}{|l|c|}
\hline Asociación & Altura (m) \\
\hline Teca + Cacao & $2,58 \mathbf{a}$ \\
\hline Fernán Sánchez + Cacao & $2,67 \mathbf{a}$ \\
\hline Promedio general & $\mathbf{2 , 6 2}$ \\
\hline Error estándar & $\mathbf{1 , 3 7}$ \\
\hline
\end{tabular}

La altura promedio del cacao obtenido en este estudio $(2,62 \mathrm{~m})$ fue inferior a los reportados por UICYT (1999), que registró una altura de 3,55 m a los cinco años de edad, en un ensayo de cacao CCN-51 asociado con cuatro especies forestales.

\section{Correlación entre la edad de las especies con el DAP y la altura total}

Al relacionar el promedio del DAP con la edad, las dos especies forestales prestaron una fuerte correlación de tendencia positiva, con $r=0,98$ para la teca y $r=0,95$ para fernán sánchez (Figura 1).

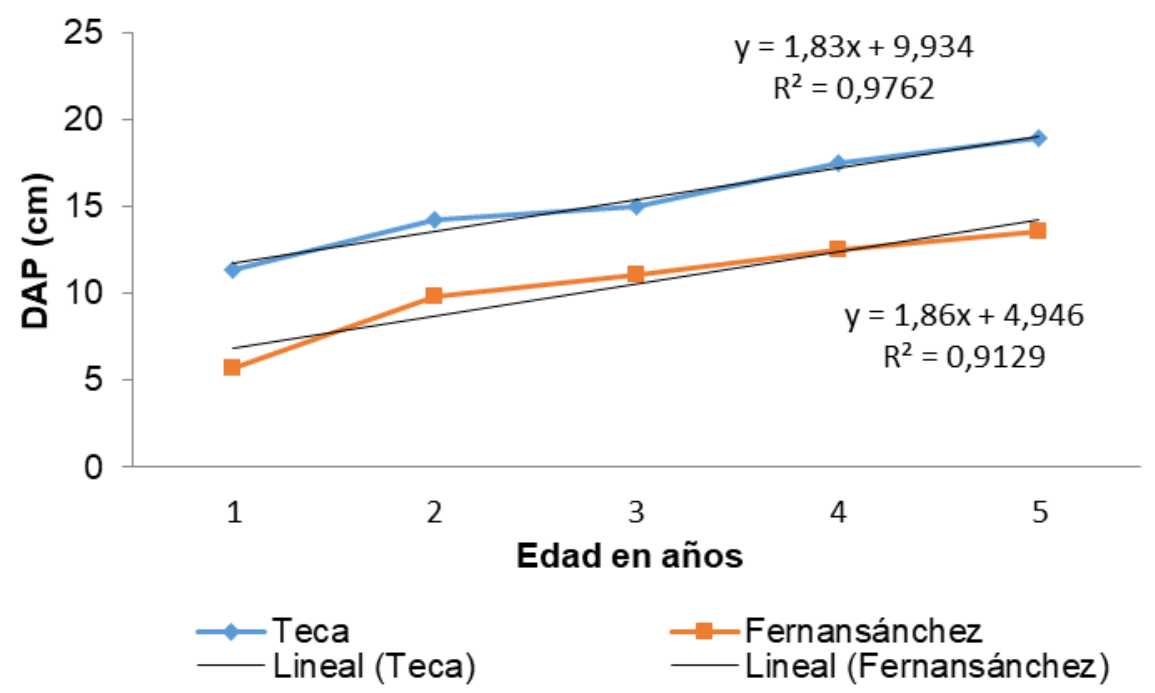

Figura 1. Correlación entre la edad y DAP de teca y fernán sánchez. UTEQ, 2013. 
Al relacionar el promedio de la altura total con la edad, las dos especies forestales prestaron una fuerte correlación de tendencia positiva, con $r=0,97$ para la teca y $r=0,99$ para fernán sánchez (Figura 2).

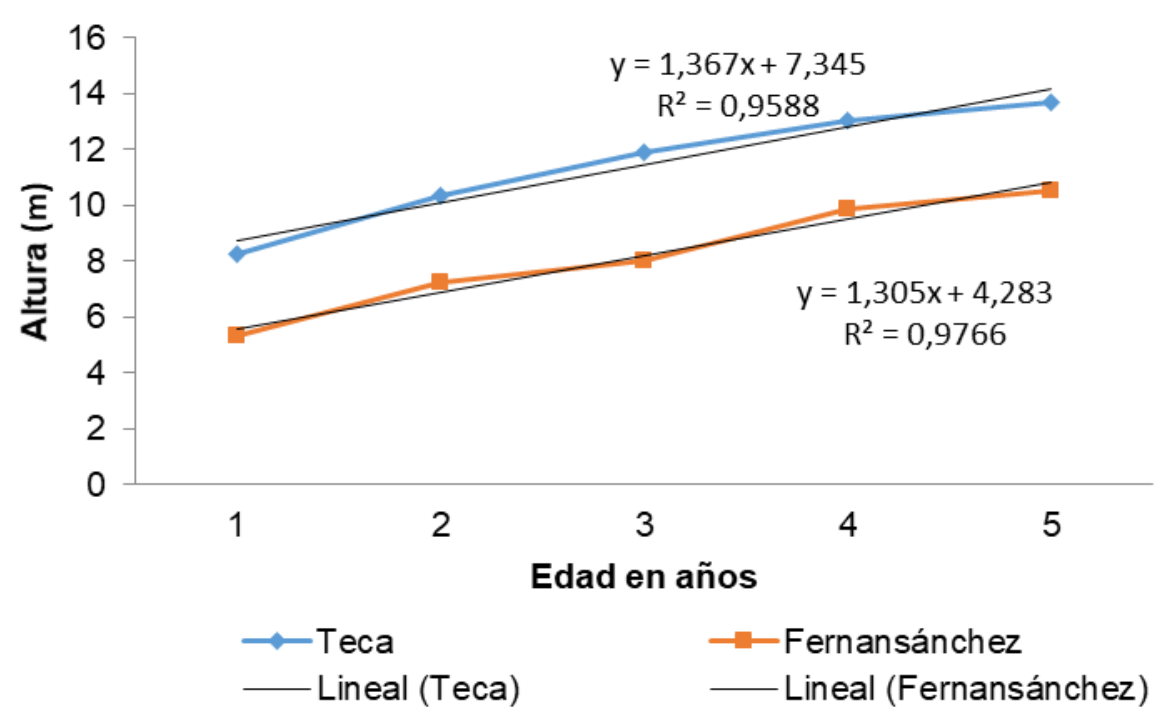

Figura 2. Correlación entre la edad y altura de teca y fernán sánchez. UTEQ, 2013.

La alta intensidad de asociación obtenida en la correlación del DAP y altura con la edad de la teca y fernán sánchez, sugiere que estas dos especies tienen un ritmo de crecimiento rápido durante los primeros años.

\section{Conclusiones}

A los cinco años de edad, la teca obtuvo los mayores promedios en diámetro y altura; además, los mayores promedios en Incremento Medio Anual (IMA) del diámetro y la altura se registraron en la teca.

A los cinco años de edad, la teca obtuvo los mayores promedios en área basal y volumen; También los mayores promedios en IMA de área basal y volumen se obtuvieron en la teca.

La altura promedio del cacao obtenido en los dos sistemas de asociación fue similar, es decir que las especies forestales no influyeron en la altura del cacao.

La asociación del DAP y la altura con la edad, en las dos especies, presentó una fuerte correlación, lo cual sugiere que hay un alto grado de interdependencias entre las variables relacionadas. 


\section{Referencias Bibliográficas}

Almeida, C.; Willy, M.; Sena-Gomes, A.; Matos, P. (2002). Sistemas agroflorestais como cacaueriro como alternativa sustantável para uso en áreas desmatadas, no estado de Rondônia, Brasil. Agrotrópical. 14 (3): 109-120.

Budowski, G. (1993). Agroforestería: Una disciplina basada en el conocimiento tradicional. Revista Forestal Centroamericana. 2 (3): 14-18.

Chaves, E. y Fonseca, W. (2004). Ensayos de aclareo y crecimiento en plantaciones de teca (Tectona grandis L.f.) en la Península de Nicoya, Costa Rica. Instituto de Investigación y Servicios Forestales (INISEFOR) Universidad Nacional, Heredia, Costa Rica. 174 p.

Duguma, B.; Gockowski, J.; Bakala, J. (1999). Desafíos biofísicos y oportunidades para el cultivo sostenible de caco (Theobroma cacao L.) en sistemas agroforestales de África Occidental y Central. Agroforestería en Américas 6 (22): 12-15.

Martinez, A. y Enríquez, G. (1981). La sombra para el cacao. Turrialba, Costa Rica, CATIE. Serie Técnica, Boletín Técnico No. 5.93 p.

Unidad de Investigación Científica y Tecnológica. (1999). Longitud de tallo y raíz en la brotación de seudoestacas de Tectona grandis L (teca). Informe Técnico anual. Universidad Técnica Estatal de Quevedo. Quevedo, Ecuador. 38 p.

Unidad de Investigación Científica y Tecnológica. (2002). Análisis de sistemas agroforestales con Coffea arabica en la zona del cantón Quevedo. Informe Técnico anual. Universidad Técnica Estatal de Quevedo. Quevedo, Ecuador. 28 p. 